INNOVATIONS IN PRIMARY CARE

\title{
Development and Implementation of a COVID-19 Respiratory Diagnostic Center
}

\author{
Amir Barzin, DO, MS ${ }^{1}$ \\ David Alain Wobl, $\mathrm{MD}^{2}$ \\ Timotby P. Daaleman, DO, MPH ${ }^{1}$ \\ 'Department of Family Medicine, University of North Carolina at \\ Chapel Hill, Chapel Hill, North Carolina \\ ${ }^{2}$ Division of Infectious Diseases, Department of Medicine, Univer- \\ sity of North Carolina at Chapel Hill, Chapel Hill, North Carolina \\ Ann Fam Med 2020;18:464. https://doi.org/10.1370/afm.2558.
}

\section{THE INNOVATION}

In response to the COVID-19 pandemic, respiratory diagnostic centers (RDCs) have emerged as a health service model that offers symptom screening and provides diagnostic testing for patients.

\section{WHO \& WHERE}

Primary care and outpatient medical directors as well as hospital leadership at the University of North Carolina Health Care System (UNCHCS) worked collaboratively to design and implement the University of North Carolina (UNC) RDC.

\section{HOW}

UNCHCS provided logistical support and resources during the design phase and convened a governance team of a nursing staff lead, physician staff lead, incident commander, medical director, and UNCHCS administrator. Guiding design principles included a patient-centered experience utilizing information technology to communicate results, and efficient use of personal protective equipment (PPE) for staff. We developed a "drivethrough" model, allowing assessment in personal vehicles. For the physical supporting structure, we used large, reception-style weatherproof tents. The referral process utilized the existing UNCHCS call center as a centralized hub to determine screening eligibility based on clinical symptoms as well as national and state testing guidelines. The call center scheduled an RDC appointment for eligible patients using the UNCHCS electronic

Conflicts of interest: authors report none.

\section{CORRESPONDING AUTHOR}

Timothy P. Daaleman, DO, MPH Department of Family Medicine University of North Carolina at Chapel Hill

Campus Box 7595

590 Manning Drive

Chapel Hill, North Carolina 27599-7595

tim_daaleman@med.unc.edu health record system. Patients arrived in a designated parking area and were directed through a series of stations; those presenting without appointments were directed to a screening area. All patients completed an intake form that included symptoms, travel history, confirmed COVID-19 contacts, and health care employment. The first station determined presence of acute respiratory symptoms of cough or shortness of breath. Symptomatic patients moved to the next station while asymptomatic patients received counseling and were discharged. Screeningeligible patients moved to the testing station where 1 staff member confirmed exposure history and symptoms and another obtained a nasopharyngeal swab for influenza and respiratory syncytial virus (RSV) testing with a reflex to COVID-19 if these were negative. After sampling, the patient was discharged with an information packet regarding testing notification, symptom management, self-isolation, and parameters for clinical followup. Patients presenting with more severe symptoms were moved to an assessment area and underwent a comprehensive assessment by a physician; patients appearing seriously ill were transferred to the emergency department. Over a 10-day period after the RDC was launched, a total of 1,074 patients were tested; 43 (4.08\%) were positive for COVID-19, 53 for influenza, and 25 for RSV. No RDC staff reported respiratory symptoms or required testing in this timeframe.

\section{LEARNING}

Several lessons were learned from launching the RDC. First was the importance of securing and monitoring the supply chain, particularly for PPE and laboratory materials. A second was to select a physical structure that could support the RDC model. We considered on-site trailers but chose reception-style tents and invehicle testing due to the capacity for high-throughput symptom assessment, targeted testing, and safe and efficient use of PPE. Infection prevention guidelines were foundational and pushed us to regularly review patient contact protocols and the use of PPE. Daily leadership huddles provided the forum to review the activities of the prior day, identify gaps, and promote new ideas to improve efficiency and workflows. Finally, we adapted our staffing model by a daily monitoring of patient volume, time analysis of throughput, and rightsizing staff for each station. This resulted in a reduction of our estimated staffing needs by $50 \%$ in 1 week without decreasing throughput.

To read or post commentaries in response to this article, see it online at https://www. AnnFamMed.org/content/18/5/464.

Key words: rapid testing; COVID-19; respiratory diagnostic center

Submitted April 2, 2020; accepted April 17, 2020. 